\title{
Traduire
}

Une autre perspective sur r tr traduction

Revue française de la traduction

$235 \mid 2016$

Luxe, mode... et traduction !

\section{Le luxe à travers les yeux du traducteur}

\section{Chiara Tampieri}

\section{(2) OpenEdition}

Journals

Édition électronique

URL : https://journals.openedition.org/traduire/838

DOI : $10.4000 /$ traduire.838

ISSN : 2272-9992

\section{Éditeur}

Société française des traducteurs

\section{Édition imprimée}

Date de publication : 1 décembre 2016

Pagination : 27-37

ISSN : 0395-773X

\section{Référence électronique}

Chiara Tampieri, «Le luxe à travers les yeux du traducteur », Traduire [En ligne], 235 | 2016, mis en ligne le 01 décembre 2018, consulté le 06 juillet 2021. URL : http://journals.openedition.org/traduire/838 ;

DOI : https://doi.org/10.4000/traduire.838 


\section{Le luxe à travers les yeux du traducteur}

\section{Chiara Tampieri}

Au cours de ces dernières années, j'ai appris à apprécier ce qui est beau, à aimer cet univers souvent riche en contradictions et à en comprendre le langage. Art, artisanat, science et technologie se mélangent dans ce domaine aussi varié qu'exigeant. Permettez-moi de vous en donner un petit aperçu.

\section{L'industrie du luxe dans le monde : un marché porteur pour la traduction}

Avec 1200 milliards de dollars de ventes en 2015, le luxe est un secteur dynamique qui occupe une place prépondérante dans l'économie mondiale.

La Chine, la France, l'Italie, I'Espagne, la Suisse, le Royaume-Uni et les États-Unis comptabilisent $84 \%$ des 100 plus grandes entreprises de produits de luxe. La France compte à elle seule 10 groupes dans le top 100, représentant 23,5\% des ventes de produits de luxe de ce palmarès. L'Hexagone se positionne comme le pays qui regroupe les entreprises de luxe les plus importantes : le trio de tête du luxe français représente $78 \%$ du total de ce marché en France.

Les attaques terroristes en Europe ont fortement ralenti le tourisme et les prévisions de croissance pour 2016 fluctuent selon les zones géographiques. En dépit de ce léger ralentissement, le secteur du luxe continue à se porter plutôt bien et à rester l'un des marchés mondiaux les plus attractifs.

Très apprécié aux quatre coins du monde, le luxe est international, n'a pas de frontières et se prête ainsi très bien à la traduction. C'est un domaine porteur pour les linguistes qui travaillent déjà pour des marques de prestige et une excellente opportunité pour tous ceux qui souhaiteraient s'orienter vers cette spécialisation. 
La diversité des secteurs où le luxe est présent (biens personnels, loisirs, gastronomie, immobilier, etc.) fait sa grande richesse. Traduisant dans leur langue les arômes d'un champagne ou les complications d'une montre de haute horlogerie, les traducteurs travaillant pour l'industrie du luxe ne sont pas interchangeables : s'ils partagent la même sensibilité, ils se seront forgé au cours de leur carrière des spécialisations bien définies.

\section{Luxe et numérique : de nouvelles opportunités pour les traducteurs}

Ce secteur historique, aujourd'hui en pleine maturité, est pourtant amené à se plier aux changements sociétaux, techniques et écologiques. Les marques mondiales de luxe doivent se remettre continuellement en question afin de satisfaire les nouvelles attentes des consommateurs.

Parmi les bouleversements qui ont touché le secteur, le plus marquant reste sans doute l'arrivée d'internet. Si l'industrie du luxe a longtemps hésité à investir le numérique, celui-ci est désormais au cœur de toute stratégie. De la conception des produits à la vente en boutique en passant par la promotion ou la relation client, le numérique a changé les pratiques des grandes marques.

Aujourd'hui, le consommateur de produits de luxe est en train d'évoluer. II ne recherche plus seulement la rareté et la qualité d'un bien, mais aussi les relations et les émotions que lui procure son achat. En s'offrant un produit, il compte vivre une expérience unique, virtuelle ou réelle, et les marques de luxe doivent être en mesure de répondre à ses attentes. La relation client devient ainsi une clé de différenciation pour ces marques. En amplifiant et en multipliant les canaux de communication, l'univers du numérique se révèle un moyen formidable pour nourrir cette relation.

Les entreprises de luxe peuvent utiliser le web pour partager des contenus qui permettent de comprendre l'histoire, les méthodes ou la philosophie de la marque. Certaines proposent des reportages photo ou des vidéos sur les réseaux sociaux ou leurs plateformes institutionnelles, voire sur des mini-sites dédiés. Ces nouveaux supports peuvent également accueillir des reportages sur l'histoire d'une maison, des interviews de ses créateurs ou de ses artisans.

Les mêmes entreprises développent également leur présence sur les réseaux sociaux avec des objectifs divers : partager l'univers de la marque, diffuser l'actualité en avant-première, gérer la relation client et vendre leurs produits. Elles comprennent que des contenus de qualité sont source de valeur, que ceux-ci ont des conséquences directes sur l'e-réputation de l'entreprise et de ses marques et peuvent avoir une influence sur le succès de ses politiques commerciales.

Ce phénomène récent a amené les traducteurs à travailler sur une multitude de supports nouveaux. Mini-sites, sites de e-commerce, blogs, pages Facebook et Twitter, mailings, newsletters, sous- 
titres de films sur les chaînes YouTube ne sont que quelques exemples des nouveaux contenus proposés désormais par les entreprises de luxe.

La traduction de ces contenus peut demander des services linguistiques annexes : par exemple, la vérification que la traduction s'affiche correctement sur les écrans d'ordinateur et de supports mobiles (smartphones et tablettes), la recherche de mots-clés à l'aide de programmes tels que Google Adwords et leur incorporation dans la traduction afin d'optimiser le référencement naturel d'un site.

Dans le secteur de la mode, l'image a souvent un rôle primordial en communication. C'est pour cette raison qu'Instagram est devenu le réseau social de prédilection des marques de mode. La parole occupe ici une position secondaire mais reste importante. Représentée par de courtes didascalies, elle se doit d'être juste et percutante.

À ces nouveaux supports s'ajoutent bien évidemment les documents classiques tels que les catalogues, lookbooks, dossiers de presse, magazines, beaux livres et supports de formation interne. Ces derniers se transforment dans certains cas en véritables plateformes d'e-apprentissage offrant des activités pédagogiques, des vidéos, des animations en 2D ou 3D et des quiz pour évaluer ou comparer les connaissances des collaborateurs. Ces contrôles de connaissances peuvent même prendre la forme de jeux-concours avec un prix à la clé.

Malgré les spécificités de chaque support, l'intervention du traducteur au service de l'industrie du luxe reste cependant intrinsèquement la même : bien saisir l'univers de la marque afin d'adapter le contenu au contenant et au destinataire. À la manière d'un journaliste, le traducteur doit mener sa petite enquête et se poser quatre questions élémentaires : "Qui ? Quoi ? Où ? Quand? ". Allons donc accompagner cet enquêteur tout au long de son voyage et ainsi réfléchir à sa démarche.

\section{Connaître son client pour mieux le servir}

Notre " envoyé spécial " va tout d'abord s'imprégner de l'univers de la marque, connaître son histoire et ses éléments distinctifs afin de les identifier et les retranscrire.

Les marques de luxe se sont forgé une identité forte et clairement définie pour révéler leurs valeurs au travers des produits qu'elles proposent. La valeur communiquée par la marque permet de différencier un produit par rapport à un autre. Face à une concurrence accrue, une marque de luxe doit revêtir une identité à la fois forte, distinctive et cohérente, offrir en tout temps et en tout lieu un visage homogène. Car les maisons de luxe ne cherchent pas à répondre à un besoin mais à construire et à proposer un univers et une identité qui soient suffisamment attractifs pour interpeller le consommateur. 
Dans le travail du traducteur, cela va nécessiter la mise en place d'un guide de style et la création d'un glossaire. Le guide de style contiendra des règles typographiques, d'orthographe, de ponctuation, des préconisations portant sur l'utilisation des guillemets, des accents, des traits d'union, des caractères majuscules, des chiffres et des vocables étrangers. II pourra également inclure des indications concernant le style et le ton à utiliser.

En matière d'utilisation de majuscules, des règles spécifiques s'appliquent aux noms des marques et des produits. On aura par exemple : "Stella McCartney ", " adidas ", "L'Oréal ", etc. La traduction des couleurs demande également une attention particulière. Le spectre chromatique n'est pas découpé de la même façon dans les différentes langues, ce qui entraîne des hésitations quant au nom de la couleur - par exemple, il est parfois difficile de trancher entre crème, beige clair et écru. De plus, les couleurs peuvent faire référence à un nom commun et à un champ sémantique qui peuvent varier d'une langue à l'autre. Si « blanc cassé " peut être traduit littéralement en italien par bianco sporco, on évitera d'utiliser l'adjectif sporco qui signifie "sale ". Pour une marque prestigieuse dans le domaine de la mode ou de la beauté et, avec le consentement du client, on parlera plutôt de bianco antico " blanc ancien " ou avorio " ivoire ", quitte à s'éloigner légèrement du champ chromatique initial.

Dans l'idéal, la traduction des couleurs devrait toujours se faire en concertation avec le client. Très souvent, ce dernier fait le choix de ne pas traduire certaines nuances (ou tout du moins les adjectifs qui les qualifient) car celles-ci sont considérées comme distinctives de la marque ou d'une collection. Chez la maison Fendi on retrouve par exemple dans la version italienne nero powder, rosa plaster, giallo alchemy, saddler et giallo Fendi.

Le même discours s'applique aux mots et expressions d'origine étrangère. Le monde du luxe adore les emprunts, le secteur de la mode en étant particulièrement friand. II suffit de regarder l'édition italienne du dernier numéro de n'importe quel grand magazine. Les titres des articles restent strictement en langue étrangère, essentiellement l'anglais, et les articles regorgent de néologismes et d'emprunts.

D'après une étude publiée sur le portail de linguistique italienne treccani.it, et menée sur 700 textes journalistiques de mode publiés entre 2012 et 2013, on relève 4,67\% d'emprunts non adaptés, soit dix fois plus que dans l'italien employé dans d'autres contextes.

Chaque saison la mode donne naissance à de nouvelles tendances, coupes et styles qu'il faut ensuite nommer. Les emprunts peuvent définir aussi bien les styles (lingerie rock, sporty style, rétro-chic, lady-like, new wave, bling-bling), les coupes (cropped, boxy, flare, boyfriend, skinny), les types de vêtements (shorts, tankini, jeggings) ou de tissus (denim dirty, denim destroy).

Si certains mots désignent des objets tout à fait nouveaux pour lesquels il serait difficile de trouver un terme italien approprié, il est néanmoins évident que dans beaucoup de cas on a affaire à des emprunts injustifiés. Par exemple, la langue italienne possède déjà un équivalent aux 
anglicismes suivants : fashionista (modaiola), fashion (moda), must-have (indispensabile), look (aspetto), shopping (acquisti), brand (marchio), loose (ampio), slim (aderente), make-up (trucco), etc.

Parfois, les emprunts de l'anglais et du français sont utilisés tout simplement comme des synonymes à employer suivant le ton et le type de lecteur. On peut oser l'hypothèse que ces mots deviennent valorisants en italien grâce à leur air de raffinement, d'exotisme et aux valeurs connotatives dont sont dépourvues leurs analogies italiennes. Ces emprunts créent un sociolecte spécifique à une partie de la population et sont l'expression d'une identité linguistique et culturelle partagée.

La connaissance de la marque, de ses valeurs, de son public cible et de l'image qu'elle veut véhiculer devient ainsi essentielle pour aider le traducteur à se frayer un chemin entre purisme et assimilation.

En s'intégrant dans la langue italienne, les emprunts peuvent subir des modifications et des adaptations. Le traducteur doit absolument être au courant de ces phénomènes afin d'éviter les surtraductions ou les traductions trop littérales. C'est le cas de termes anglais comme bomber jacket et clutch bag pour lesquels, en italien, on assiste à un phénomène de réduction. On utilisera donc tout simplement bomber et clutch.

Ces dernières années, l'apport traditionnel du français à la langue du luxe et de la mode s'est atténué. Résistent les emprunts qui appartiennent au lexique traditionnel comme "atelier ", " couturier ", " couture ", " ton sur ton " et " prêt-à-porter ", ainsi que les technicismes qui décrivent des matières et des traitements spécifiques, tels que " chiffon ", " voile ", " satin ", " crêpe ", " pied-de-poule ", " godet ", " matelassé ", " plissé ".

Or, le français peut parfois se révéler trompeur pour le traducteur d'italien en raison des fauxamis, des termes français qui sont rentrés dans le langage italien de la mode, mais avec une signification différente. Le terme "mélange " en est un bon exemple. En français il est utilisé pour indiquer une composition, un mélange de matières, ce qui correspond à l'italien misto. Dans la langue de Dante, par contre, le gallicisme " mélange " est l'équivalent du français " chiné ", se référant à un fil teint en masse avec différentes couleurs pour obtenir un effet bigarré après tissage.

L'anglais de la mode fait également appel au français pour ce qui est du lexique technique. Le risque est que ces mots se modifient peu à peu à la manière du téléphone arabe : il m'est arrivé par exemple de trouver en anglais molton avec la signification de " molleton " malgré le fait que l'anglais dispose de son propre équivalent (fleece), ce qui oblige les traducteurs depuis l'anglais à avoir des connaissances du français de la mode et à redoubler d'attention.

Parfois certains vocables du langage courant sont adoptés par les marques pour désigner une nouvelle collection ou un produit. C'est le cas de la collection d'accessoires Gucci Signature, de 
la ligne de sacs de la même marque Bright Diamante, du ruban Web, etc. Le traducteur devra prêter une attention particulière à ces termes qui seront laissés tels quels ou traduits suivant le choix de la marque.

Cela est également valable pour les slogans, les dictons et les aphorismes que les marques peuvent choisir de laisser en langue étrangère (et accompagner éventuellement d'une traduction). Quelques exemples tirés du site internet d'une grande maison horlogère : Seaside Living in the Hamptons, Life is about moments.

Si la marque de luxe doit à la fois incarner une personnalité forte, distinctive et cohérente en offrant un visage homogène, nous assistons depuis quelque temps à un changement dans l'identité du consommateur. En effet, le client est de plus en plus " global " et devient un vrai consommateur caméléon qui achète plusieurs marques en même temps. Face à cette mutation, les marques ont réagi et ont travaillé sur des identités plus dynamiques.

Nous assistons donc à la mise en vente de produits et de lignes de différentes gammes de prix : du luxe accessible à l'extra-luxe en passant par une offre sélective. Les maisons de luxe peuvent aussi décider de segmenter leur offre en créant des sous-marques de leur marque plus prestigieuse, destinées à une clientèle plus large et/ou plus jeune.

Quelles sont les implications de ce phénomène pour le traducteur? Prenons par exemple le cas de la marque Alexander McQueen et de sa sous-marque McQ, toutes deux appartenant à un groupe géant du luxe.

McQ représente la vision plus contemporaine, plus urbaine et plus jeune de la marque. Elle s'inspire de l'esprit rebelle et streetstyle de son créateur en y ajoutant une interprétation plus brute des codes de la maison.

Cette marque se positionne dans le créneau des vêtements et accessoires contemporains et accessibles; des produits inspirés par la culture de la rue et par la scène artistique et musicale britannique qui incarnent une beauté nonchalante, une attitude anticonformiste et la simplicité extravagante de la marque.

Le discours change si on considère la marque principale, Alexander McQueen, réputée pour ses designs conceptuels, son identité forte et sa créativité débridée. Connue pour ses robes spectaculaires, ses talons aiguilles vertigineux et ses matières précieuses, cette griffe est plus proche de l'univers de la haute couture que de celui du prêt-à-porter. Elle s'adresse à un public de 25 à 55 ans, cultivé et cosmopolite, sophistiqué et aisé. Ses produits haut de gamme sont un concentré de très haute qualité, d'innovation, d'émotion et du plaisir offert par la mode.

À la manière d'un couturier, le traducteur devra être capable de tailler un texte sur mesure pour ces deux marques et leur clientèle. Si pour McQ il pourra utiliser un langage jeune, brut et branché (feu vert donc aux néologismes et aux anglicismes), dans le cas d'Alexander McQueen 
l'écrit devra s'avérer plus raffiné, évocateur et rêveur, ceci toujours dans un souci de fidélité au texte d'origine.

En s'adressant aux clients d'une marque de luxe, on s'adresse également à une population restreinte et très impliquée, à une sorte de tribu. Le traducteur doit être capable de comprendre, interpréter et transmettre l'image linguistique de cette marque au public ciblé. Pour ce faire, il doit maîtriser l'art de la narration et favoriser une communication personnelle et personnalisée.

\section{Le produit au cœur du processus de traduction}

La connaissance profonde du produit est également essentielle dans le travail du traducteur. De l'industrie du textile à la gastronomie en passant par la maroquinerie, la joaillerie, l'horlogerie ou la parfumerie, sans oublier l'hôtellerie et l'immobilier de prestige, l'industrie du luxe regroupe divers secteurs et catégories de produits.

Si la langue du luxe peut avoir des caractéristiques communes, il ne faut pas oublier que chaque produit est différent et demande des connaissances spécifiques. II est souvent le fruit d'une recherche poussée, de matières nobles et de techniques innovantes. Le traducteur doit être en mesure de transmettre par les mots le savoir-faire qui se cache derrière un tissu, une matière mais également partager sa dimension sensorielle.

Le défi s'avère de taille dans un domaine comme celui de la haute horlogerie, où tradition et innovation se rencontrent. Une montre de luxe est à la fois un instrument de haute précision et une œuvre d'art. Elle réunit des techniques industrielles de pointe et des gestes précis, fruits d'un savoir-faire ancestral.

Qu'il s'agisse de traduire la fiche technique de la montre la plus compliquée au monde créée en 2015 par Vacheron Constantin (elle comporte 57 complications, 2826 composants et une dizaine de brevets en cours de dépôt) ou la description des métiers d'art de Cartier appliqués à l'horlogerie (granulation, filigrane, émail champlevé, grisaille pâte d'or ou émail plique à jour, pour n'en nommer que quelques-uns), le traducteur doit bien connaître son sujet et avoir toujours le mot juste et précis. Dans ce secteur en constante évolution, nous ne cessons jamais d'apprendre et en cas d'impasse, il est essentiel de recourir à l'aide d'un expert, voire du client.

Dans la présentation d'une montre de luxe, nous trouverons souvent des contenus très évocateurs qui permettent d'illustrer l'univers du produit, de créer une ambiance et de s'adresser à une audience spécifique. 
En général, I'horlogerie, et d'autant plus la haute horlogerie, cible une clientèle d'âge mûr et de très haut niveau. Cela se reflète dans la complexité des structures grammaticales, dans des phrases plus longues et dans un vocabulaire plus technique et moins contaminé par les emprunts.

\section{Le contenant pour le contenu}

L'étape suivante pour le traducteur consiste à réfléchir au type de support sur lequel sera publié le texte à traduire. Des fiches produits seront denses, synthétiques et explicatives alors que d'autres contenus auront un caractère davantage publicitaire et évocateur. Le ton choisi pour un tutoriel de maquillage sur YouTube ne sera pas le même que celui utilisé pour communiquer à la presse l'ouverture d'une nouvelle boutique monomarque. Il est important de penser au média visé et de choisir la bonne tonalité, tout en restant fidèle aux objectifs de communication de la marque.

Une aide précieuse à cet égard sera le brief, document qui réunit les informations, les objectifs et les demandes du client dûment adaptés à un projet de communication(1). Pour chaque nouvelle traduction, le client devrait, si possible, fournir le brief du texte pour permettre au traducteur de connaître le lectorat, le support prévu et le registre souhaité par le client.

Prenons le cas de la newsletter, document envoyé périodiquement par une entreprise de luxe aux personnes qui ont fait la démarche de s'y inscrire. Ces contenus peuvent être multiples et en rapport avec toute l'actualité de la maison. La newsletter est un outil de marketing essentiel pour entrer en contact avec les clients et les inviter à l'action (visiter un site internet, participer à un événement, acheter un produit, etc.), afin de s'en rapprocher de manière plus individuelle.

Face à ce type de contenu le traducteur est amené à se poser la question quasi shakespearienne suivante : "Être ou ne pas être - formels ? ". La langue italienne offre différentes possibilités : le tu, qui correspond au tutoiement, le lei équivalent du vouvoiement, le "vous " du pluriel voi, et l'infinitif.

Si, en Italie, la communication web (sites internet, newsletters, réseaux sociaux) préfère en général le tutoiement, même dans l'univers du luxe), le choix final du positionnement par rapport aux lecteurs revient au client.

Certaines marques continuent à privilégier le vouvoiement et les formules impersonnelles dans la version italienne de leurs newsletters. D'autres maisons de luxe recherchent plus de proximité

(1) Pour une analyse détaillée du brief en italien, voir http://www.mestierediscrivere.com/articolo/brief. 
avec leurs lecteurs en choisissant le tutoiement pour leur newsletter tout en préférant l'utilisation de l'infinitif et du vouvoiement sur leur site internet. Souvent on retrouve une utilisation incohérente des formules de politesse, ce qui est révélateur d'un travail de traduction à plusieurs mains, qui manque malheureusement d'homogénéisation.

Pour être efficaces, les newsletters doivent être adressées aux bons destinataires et au bon moment. Grâce aux connaissances socioculturelles des pays cibles, les traducteurs peuvent jouer un rôle précieux de conseil auprès des marques.

Ils peuvent, par exemple, être amenés à traduire des messages liés à des fêtes qui ne sont pas connues dans le pays cible ou qui ne sont pas célébrées à la même date. C'est le cas notamment de la fête des mères ou de la fête des pères dont la date varie d'un pays à l'autre. En l'espèce, le traducteur devra alors conseiller de modifier le texte et les dates d'envoi afin de les adapter au public ciblé.

S'il s'agit d'une fête qui n'est pas célébrée dans le pays de destination du message, il appartiendra toujours au traducteur de le signaler à son client, lequel pourra décider de la suite à donner. Ainsi, en Italie, nous ne fêtons pas Thanksgiving, toutefois il peut être intéressant pour la marque de faire découvrir cette fête à ses clients dans la mesure où elle serait en relation avec l'identité et l'univers de la maison.

Si nous nous intéressons aux supports publicitaires d'une manière plus générale, nous remarquerons immédiatement un rapport texte/image qui penche en faveur de cette dernière, avec des illustrations très riches et des textes d'accompagnement se résumant au nom de la marque ou, tout au plus, au nom de la collection ou à un slogan.

Ces textes répondent cependant à des règles précises, notamment en France, en ce qui concerne l'utilisation des termes d'origine étrangère. Alors que la loi du 4 août 1994 (cf. article d'Agnès Portais dans ce même numéro, page 15), appelée loi Toubon, impose l'utilisation de la langue française dans le domaine de la communication marketing et commerciale ainsi que dans celui des messages publicitaires et aux différents documents commerciaux (conditionnement, garantie, facture, etc.), elle n'interdit pas l'utilisation d'expressions étrangères, mais, dans ce cas, les termes utilisés doivent être traduits sur le document ou support. Des lois de ce type n'existent pas en Italie et aucune protection n'est octroyée à la langue de Dante. En l'occurrence, en feuilletant un magazine de mode, un lecteur italien demeurerait plus interloqué par la vue d'un renvoi explicatif de bas de page que par la lecture d'un slogan en anglais, même s'il n'en comprend pas le sens.

Dans ce cas, la liberté d'action du traducteur est très limitée, la décision de traduire ou pas revenant directement au client final. Le plus souvent les noms des produits ou les slogans restent dans la langue d'origine. Est-ce que le lecteur comprendra à partir d'une photo que le nouveau Synchro Skin Lasting Liquid Foundation de Shiseido est un fond de teint ? Apparemment oui. 


\section{La traduction contre la montre}

Entre lancements de produits, défilés, expositions et salons, le calendrier du luxe est riche en événements et pas un mois ne passe sans grand rendez-vous.

Considérée comme l'un des moments forts du calendrier de la mode, la Fashion Week est un événement durant approximativement une semaine et qui a lieu deux fois par an pour permettre aux stylistes et aux grandes maisons de présenter leurs dernières collections de prêt-à-porter et de haute couture.

Les quatre plus importantes, souvent appelées les Big Four par les médias anglo-saxons, sont celles qui se tiennent dans les capitales de la mode : Paris, Milan, New York et Londres. À partir des années 2000, les Fashion Week se sont également étendues à d'autres parties du monde. Certaines semaines de la mode présentent des produits spécifiques comme les maillots de bains à la Fashion Week de Miami.

Traditionnellement, les semaines de la mode sont en décalage avec la réalité saisonnière des produits présentés et le défilé a lieu environ 6 mois avant que la collection n'arrive en boutique. La communication dans la mode, et par conséquent sa traduction, suit ce modèle en deux phases. Le traducteur sera sollicité avant ou pendant la " semaine ". II traduira les dossiers de presse et autres supports qui accompagnent la présentation des collections aux acheteurs en showroom et lors des défilés. II sera également amené à traduire la revue de presse d'une maison qui souhaiterait comprendre comment sa collection a été perçue par les médias étrangers. Des demandes de traduction pourront également coïncider avec la mise en boutique des nouvelles collections et les opérations marketing en magasin tel que les soldes, les fêtes de la Saint-Valentin ou de fin d'année.

Confrontés à la fréquence de renouvellement des produits chez les grandes chaînes de prêtà-porter, les marques de luxe et les créateurs ont ajouté à leur calendrier de nouvelles collections intermédiaires, appelées cruise et pre-fall, qui apportent aux clients la nouveauté qu'ils recherchent.

Certaines maisons sont allées plus loin en épousant un tout nouveau modèle économique et événementiel : le see now, buy now. Le concept consiste à rendre la collection disponible à la vente juste après le défilé. II s'agit en fait de tirer profit de l'exposition médiatique qu'offre ce dernier et de favoriser ainsi les achats impulsifs.

Une grande maison britannique, pionnière en ce sens, a présenté en septembre de cette année son premier défilé " adressé au consommateur " : les 83 modèles masculins et féminins ont tous été disponibles instantanément, juste après le défilé, dans ses boutiques ou en ligne, avec des livraisons dans plus de 100 pays. Les nouvelles campagnes publicitaires et les vitrines des boutiques ont été également dévoilées au même moment. 
La communication dans le luxe devient ainsi de plus en plus rapide et exige une grande disponibilité et réactivité de la part du traducteur. Dans des délais imposés et souvent très serrés, celui-ci doit associer la précision et la rigueur d'une traduction technique à l'excellente qualité rédactionnelle requise par la traduction éditoriale. Un défi qui se révèle formateur et enrichissant, jalonné d'obstacles mais aussi de belles satisfactions.

chiara.tampieri@gmail.com

Traductrice et interprète installée à Lyon, Chiara Tampieri traduit du français et de l'anglais vers l'italien. Après une expérience de trois ans en agence, elle décide en 2010 de s'installer à son compte. C'est le début d'un parcours de spécialisation qui va la conduire à travailler pour de nombreuses marques dans l'univers du luxe, de la mode et de l'art de vivre, des secteurs qui sont désormais au cœur de son activité. Passionnée par les nouvelles technologies qu'elle utilise pour optimiser son travail, elle est également en charge du cours de traduction assistée par ordinateur à l'Université de Lyon 3.

\section{Bibliographie}

ATI KENT Aurélie, 2004, Les marques de luxe face à l'engouement du grand public, Grenoble, Wesford Ecole supérieure de commerce, http://www.memoireonline.com/01/06/80/m_marquesluxes-grand-public0.html, consulté le 2 octobre 2016.

CYNARSKA-CHOMICKA Barbara, 2011, "Les anglicismes récents dans le vocabulaire français de la mode ", in Romanica Cracoviensia, 11, p. 75-82.

Deloitte Financial Advisory, 2016, Global Fashion \& Luxury market: Private Equity and investors survey 2016, http://www.deloitte-france.fr/formulaire/global-fashion-luxury-private-equity2016, consulté le 2 octobre 2016.

McCRACKEN Grant David, 2008, Transformations: Identity Construction in Contemporary Culture, Bloomington, (États-Unis), Indiana University Press.

ONDELLI Stefano, "Da chic a glam: gli anglicismi alla conquista della moda italiana ", http://www.treccani.it/lingua_italiana/speciali/moda2/Ondelli.html, consulté le 2 octobre 2016.

STOKBURGER-SAUER Nicola, RATNESHWAR S. et SEN Sankar, 2012, "Drivers of consumerbrand identification ", in International Journal of Research in Marketing, 29, p. 406-418. 